\title{
Performance Analysis of SAC/Optical CDMA and WDM under a Hybrid Overlay Scheme
}

\author{
Isaac A. M. Ashour ${ }^{1}$, Student Member, IEEE, Hossam M. H. Shalaby ${ }^{2}$, Senior Member,IEEE and P Susthitha \\ Menon $^{1}$, Member, IEEE \\ ${ }^{1}$ Institute of Microengineering and Nanoelectronics(IMEN), Universiti Kebangsaan Malaysia, 43600 UKM Bangi, \\ Selangor, Malaysia \\ ${ }^{2}$ Department of Electronics and Communications Engineering, School of Electronics, Communications, and \\ Computer Engineering, Egypt-Japan University of Science and Technology (E-JUST), Alexandria 21934, Egypt \\ isaacash@eng.ukm.my
}

\begin{abstract}
This paper presents a performance analysis of both optical code-division multiple-access (OCDMA) and wavelength division multiplexing (WDM) in an overlay hybrid scheme. Modified quadratic congruence (MQC) code pulses of spectral amplitude coding (SAC) for optical CDMA is utilized as the signature address codes. This hybrid system is described, and its performance is analyzed, taking into account interferences and some types of noises. It is shown that the performance results in terms of both the bit-error rate (BER) and signal-to-noise and interference ratio (SNIR) are accepted in optical communication networks.
\end{abstract}

Keywords: Optical code division multiple access (OCDMA), spectral amplitude coding (SAC), wavelength division multiplexing (WDM).

\section{INTRODUCTION}

Recently, Optical CDMA systems have an important role in optical networks because these systems provide the users simultaneous and asynchronous access to the network with high security $[1,2]$. SAC/OCDMA spectrum can be in-band transmitted with multichannels of WDM system to get some advantages of transmission capacity and data confidentiality enhancement [3, 4]. Modified quadratic convergence (MQC) code is used as a signature address code for SAC/OCDMA system. This scheme has features such as a significant reduction in the effects of multiple access interference (MAI) and attenuation in the effects of phase induced intensity noise (PIIN) [5].

In previous studies, The WDM and SAC/OCDMA hybrid overlay system have been studied in [6, 7], where each WDM channel can employ the same set of SAC/OCDMA systems. In [3] we have investigated the feasibility of transmitting both OCDMA and WDM users on the same spectrum band. Both hybrid and non-hybrid systems have been simulated and the performance comparison is presented. Our results in [3] indicate that it is possible to achieve an acceptable performance with good data confidentiality under the hybrid scheme. The results in [3] have been only demonstrated by simulation. Theoretical analysis of the performance, however, has not been demonstrated yet for this system.

In this paper, we analyze the performance of both SAC/OCDMA-WDM systems under hybrid overlay system theoretically. Hence, the overall signal-to-noise and interference ratio (SNIR) is derived and the corresponding bit-error rate (BER), taking into consideration various types of noises and interferences. In addition, we obtain the numerical results with the effects of system parameters. The remainder of this paper is organized as follows: In Section 2 we describe the proposed system. In Section 3 we analyze the performance of the hybrid system. In Section 4, numerical results are presented for both sub-systems (SAC/OCDMA and WDM). Finally the conclusion of the paper is provided in Section 5.

\section{SYSTEM DESCRIPTION}

In the proposed system [3, 4], narrow-band WDM signals and optical broadband CDMA signals are in-band transmitted over the same spectral region. A block diagram of the hybrid WDM-SAC/OCDMA system is shown in Fig. 1. Fig. 2 (a) illustrates that the CDMA signals are spread over a band of $\Delta v$, whereas each WDM signal covers a band of $B_{w}$. In addition, the frequency spacing between WDM signals is $100 \mathrm{GHz}$ as the ITU standards [10]. So that, the maximum number of WDM channels, $N_{w}$ is $\Delta v / 100 \mathrm{GHz}$. At the OCDMA receiver side, the WDM interferences can be attenuated by using notch filters. Fig. 2 (b) shows the function of an ideal notch filter that is utilized in the proposed system.

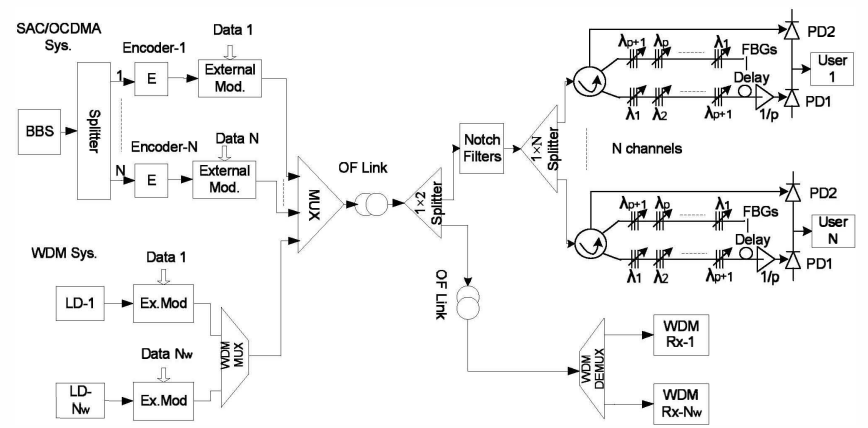

Figure 1. Block-diagram of the hybrid system.

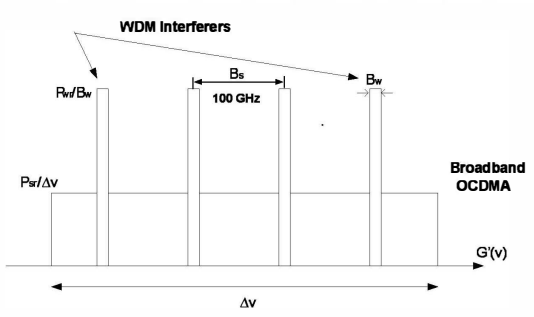

(a) 


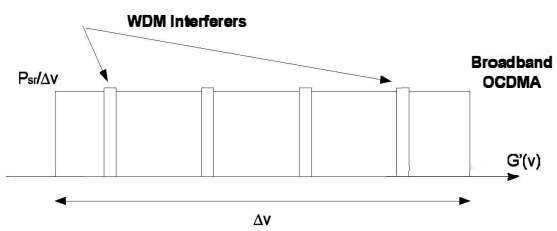

(b)

Figure 2. SAC/OCDMA and WDM- overlay signals. (a) Narrow-band signals of WDM and broad-band signals of OCDMA. (b) Function of an ideal notch filter.

The SAC/OCDMA system consists of a broadband source and point-to-point transmission channels by using star coupler. The data of each user is encoded by MQC code families $\left(p^{2}+p, p+1,1\right)$ with $p$ a prime number. The properties of MOC codes are mentioned in [5], where an MQC code is denoted by $(N, w, \lambda)$, where $N$ is the number of code words, $w$ is the code weight, and $\lambda$ is the cross-correlation. In this code the optimal number of users $K$ equals $p^{2}$.The public WDM system setup consists of CW laser sources for some channels that are modulated at specific data rate for each user. The two systems are using on-off keying modulation and are combined together over a single fiber link. At the receiver side of the system, the WDM receiver consists of a narrow band-pass filter followed by $\mathrm{O} / \mathrm{E}$ conversion. The SAC/OCDMA receiver is based on the complementary detection scheme [5], following a notch filter designed for the hybrid system.

\section{PERFORMANCE ANALYSIS}

The signals from both WDM and OCDMA channels are independently combined into the optical fiber link. The performance of both systems is limited by the multi-access interference in addition to the phase-induced intensity noise (PIIN), shot noise, and thermal noise. In this section we develop a theoretical analysis of the system under consideration.

\section{A. SAC/OCDMA Subsystem Analysis}

In this subsystem with ideal broadband light source (BLS), the assumptions and symbol definitions follow that of previous studies [5, 8-9]. In addition, the chip width $B_{c}$ of an MQC code of SAC/OCDMA is equal to the bandwidth of a WDM channel $B_{w}$. That is, $B_{c}=B_{w}$.

The received SAC/OCDMA signal, before the notch filters and OCDMA decoders, $r(v)$, is a sum of the transmitted signal, $s(v)$ and the WDM interferers, $w(v)$.

$$
\begin{gathered}
r(v)=s(v)+w(v) \\
s(v)=\frac{P_{s r}}{\Delta v} \sum_{k=1}^{K} d_{k} \sum_{i=1}^{N} c_{k}(i)\{\operatorname{rect}(i)\} \\
w(v)=\frac{P_{w r}}{B_{w}} \sum_{i=1}^{N} A_{i}\{\operatorname{rect}(i)\}
\end{gathered}
$$

where $P_{s r}$ is the effective power of a broad-band source at the receiver (there is power loss due to transmission and star coupler), $c_{k}(i)$ denotes the ith element of the kth MQC code sequence, $\mathrm{K}$ is the number of CDMA users, and $d_{k}$ is the data bit of the kth-user, $d_{k} \in\{0,1\} . P_{w r}$ is the effective power of WDM pulses at the receiver and for each $i \in\{1,2, \ldots, N\}$

$$
A_{i}=\left\{\begin{array}{l}
1 ; \text { if a WDM signal exists at the } i \text { th chip } \\
0 ; \text { else }
\end{array} .\right.
$$

Notice that $\sum_{i=1}^{N} A_{i}=N_{w}$, where $N_{w}$ is the number of WDM users.

The $u\{v\}$ is the unit step function and rect(i) is given by

$$
\begin{aligned}
\operatorname{rect}(i)=u\left[v-v_{0}-\frac{\Delta v}{2 N}(-N+2 i-2)\right] \\
-u\left[v-v_{0}-\frac{\Delta v}{2 N}(-N+2 i)\right]
\end{aligned}
$$

Therefore, equation (1) can be rewritten as follows:

$$
r(v)=\frac{P_{s r}}{\Delta v} \sum_{k=1}^{K} d_{k} \sum_{i=1}^{N} c_{k}(i)\{\operatorname{rect}(i)\}+\frac{P_{w r}}{B_{w}} \sum_{i=1}^{N} A_{i}\{\operatorname{rect}(i)\}
$$

As mentioned above, the function of the notch filters is to reject the high power of WDM interference. We suppose that the notch filters are ideal attenuated the WDM power to the level of an OCDMA signal, Fig. 2(b). That is the power spectral density (PSD) of the attenuated WDM signal equals $P_{s r} / \Delta v$.The effect of the notch filter on the OCDMA chips can be neglected as long as $N \gg N_{w}$. Therefore, the PSD of the receiving signals at PD1 and PD2 of user $l \in\{1,2, \ldots, K\}$, during one bit period can be expressed as

$$
\begin{aligned}
G_{1}(v)= & \frac{1}{p} \frac{P_{s r}}{\Delta v} \sum_{k=1}^{K} d_{k} \sum_{i=1}^{N} c_{k}(i) \overline{c_{l}}(i)\{\operatorname{rect}(i)\} \\
& +\frac{1}{p} \frac{P_{s r}}{\Delta v} \sum_{i=1}^{N} A_{i} \bar{c}_{l}(i)\{\operatorname{rect}(i)\} \\
G_{2}(v)= & \frac{P_{s r}}{\Delta v} \sum_{k=1}^{K} d_{k} \sum_{i=1}^{N} c_{k}(i) c_{l}(i)\{\operatorname{rect}(i)\} \\
& +\frac{P_{s r}}{\Delta v} \sum_{i=1}^{N} A_{i} c_{l}(i)\{\operatorname{rect}(i)\}
\end{aligned}
$$

Considering incoherent intensity noise, shot noise, and thermal noise, the combined 3 noises can be expressed as

$$
\left\langle N_{\text {Noise }}^{2}\right\rangle=\left\langle i_{\text {PIIN }}^{2}\right\rangle+\left\langle i_{\text {Shot }}^{2}\right\rangle+\left\langle i_{\text {Th }}^{2}\right\rangle
$$

The average interference photocurrent due to WDM signals

$$
\left\langle\text { Interf } .^{2}\right\rangle=\left(\mathfrak{R} \frac{P_{s r} N_{w}}{N^{2}}\left(1+\frac{1}{p}\right)\right)^{2}
$$

Then the signal-to-noise and interference ratio (SNIR) is

$$
S N I R=\frac{\left(\Re P_{s r} / 2 p\right)^{2}}{\left\langle N_{\text {Noise }}^{2}\right\rangle+\left\langle\text { Interf } .^{2}\right\rangle}
$$

\section{B. WDM Subsystem Analysis}

The second part of the analysis focuses on the WDM system performance under the hybrid scheme. Each WDM signal performance strongly depends on the peak power ratio used between WDM users and SAC/OCDMA users. We assume that the receiving peak power for all WDM users is equal. The receiving signal at a WDM demultiplexer can be written as 


$$
r(v)=\frac{P_{s r}}{\Delta v} \sum_{k=1}^{K} d_{k} \sum_{i=1}^{N} c_{k}(i)\{\operatorname{rect}(i)\}+\frac{P_{w r}}{B_{w}} \sum_{i=1}^{N} A_{i}\{\operatorname{rect}(i)\}
$$

the PSD of the PD at the $l$ th WDM receiver during one bit period can be written as

$$
f_{l}(v)=\frac{P_{s r}}{\Delta v} \sum_{k=1}^{K} d_{k} c_{k}(i)\{\operatorname{rect}(i)\}+\frac{P_{w r}}{B_{w}} \operatorname{rect}(i)
$$

the total average noise and interference, respectively:

$$
(\text { WDM noise })^{2}=
$$

$$
\begin{aligned}
B_{e w} \Re^{2}\left[\frac{P_{w r}^{2} N}{\Delta v}+\frac{P_{s r}^{2}}{N \Delta v} \frac{K}{2 p}+\frac{P_{s r} P_{w r} K}{p \Delta v}\right] \\
+2 e B_{e w} \Re\left[P_{w r}+\frac{P_{s r} K}{2 N p}\right]+\left(4 K_{b} T_{n} B_{e w} / R_{L}\right) \\
\left(\text { Interf }_{W D M}\right)^{2}=\left(\frac{\Re P_{s r} K}{2 p N}\right)^{2}
\end{aligned}
$$

and the average WDM signal:

$$
(\mathrm{WDM} \text { signal })^{2}=\left(\Re P_{w r}\right)^{2}
$$

Hence, using (13), (14) and (15), we have

$$
S N I R_{W D M}=\frac{(\text { WDM signal })^{2}}{(\text { WDM noise })^{2}+\left(\text { Interf }_{W D M}\right)^{2}}
$$

\section{NUMERICAL RESULTS}

The typical parameters used for the performance of hybrid system calculation are illustrated in Table I. We have employed this expression $B E R=0.5 \operatorname{erfc}(\sqrt{S N I R / 2})$ to obtain the performance of SAC/OCDMA subsystem in the hybrid system. Fig. 3 shows the relation between bit-error rate (BER) and the number of active users for various number of WDM channels $\left(N_{w}\right)$, with using $p=9$, the effective power is $-10 \mathrm{dBm}$ and the bit rates are $622 \mathrm{Mbps}$. The performance of this system reduces when the number of active users increases due to PIIN. It is also noticed that the performance decays with rising of WDM channels due to the effects of interferences. Therefore, as long as $N>N_{w}$ the effects of WDM interferes can be neglected. This paper emphasizes on the performance analysis of the hybrid system. The complete description of the increment in confidentiality of the hybrid system as compared to non-hybrid system has been published in [3].

The results of WDM system performance under the hybrid scheme is shown in Fig. 4, whereas the WDM system's SNIR is versus the received power, $P_{w r}$, when the effective power of SAC/OCDMA, $P_{s r}$, starts at $-20 \mathrm{dBm}$, using various values of bit rates for WDM. We can clearly see that the WDM SNIR decreases with decreasing received power and with increasing bit rates. These results show that the performance of WDM users is accepted in spite of the hybrid scheme.

TABLE I

TYPICAL PARAMETERS USED FOR CALCULATION

\begin{tabular}{l|l}
\hline Responsivity of the PDs & $\Re=0.75$ \\
\hline Electron's charge & $e=1.6 \times 10^{-19}$ \\
\hline Boltzmann's constant & $K_{b}=1.38 \times 10^{-23}$ \\
\hline
\end{tabular}

\begin{tabular}{l|l}
\hline Receiver noise temperature & $T_{n}=300 \mathrm{~K}$ \\
\hline Receiver load resistor & $R_{L}=1030 \Omega$ \\
\hline Line-width of the thermal source & $\Delta v=3.75 \mathrm{THz}$ \\
\hline $\begin{array}{l}\text { Electrical bandwidth (for } \\
\text { SAC/OCDMA) }\end{array}$ & $B_{e c}=320 \mathrm{MHz}$ \\
\hline Electrical bandwidth (for WDM) & $B_{e v}=1.29 \mathrm{GHz}$ \\
\hline Data bit rate & $622 \mathrm{Mbps}$ and $2.5 \mathrm{Gbps}$ \\
\hline Operating wavelength & $\lambda_{0}=1.55 \mu \mathrm{m}$ \\
\hline
\end{tabular}

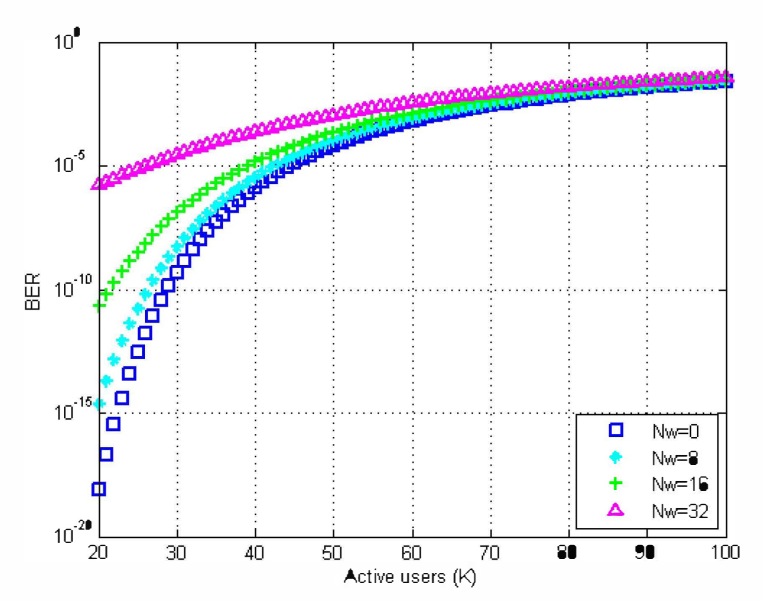

Figure 3. Number of active users versus BER at $p=9, P_{s r}=-10(\mathrm{dBm})$ and various $N_{w}$ values.

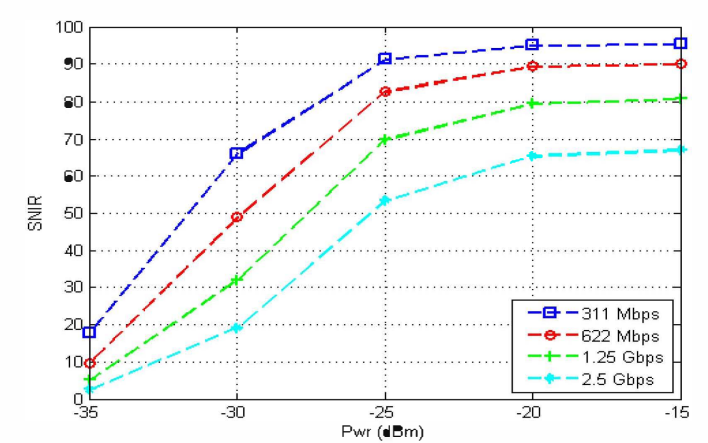

Figure 4. SNIR of hybrid WDM system vs. various received power, $P_{w r}$

\section{CONCLUSION}

We have analyzed the performance of both SAC/Optical CDMA and WDM systems in a hybrid scheme, considering the effects of intensity noise, interference, thermal noise and shot noise. The intensity noise and the interference are the main factors that limit the system performance. However, the proposed hybrid scheme makes an eavesdropper to face another challenge for intercepting and decoding the coded signal hence improving the overall security of OCDMA systems.

Acknowledgment

We would like to acknowledge Universiti Kebangsaan Malaysia for sponsoring this project under grant no UKMGGPM-NBT-090-2010 and UKM-OUP-NBT-27-119/2011. We also would like to thank the management of UKM for the fellowship grant "ZAMALAH". 


\section{REFERENCES}

[1] J. A. Salehi, "Code division multiple access techniques in optical fiber network-Part I: Fundamental principles," IEEE Trans. Commun., vol. 37, pp. 824-833, Aug. 1989.

[2] J. A. Salehi and C. A. Brackett, "Code division multiple access techniques in optical fiber network-Part II: System performance analysis," IEEE Trans. Commun., vol. 37, pp. 834-842, Aug. 1989.

[3] I. A. M. Ashour, S. Shaari, H. M. H. Shalaby, and P. S. Menon, "Investigation of in-band transmission of both SAC/OCDMA and WDM signals," SPIE Opt. Eng. 50(6), 1-7(June 2011).

[4] I.A. Ashour, S. Shaari, H.M. Shalaby, P.S. Menon, and H.A. Bakarman, "Hybrid SAC/Optical CDMA-WDM overlay system for enhancing network security," in Proc. IEEE Conf. International Conference on Photonics (ICP2010), pp. 1-4, Langkawi, Malaysia (2010).

[5] Z. Wei, H. M. H. Shalaby, and H. G. Shiraz, "Modified quadratic congruence codes for fiber Bragg-grating-based spectral amplitude coding optical CDMA systems," J. Lightwave Technol. 19(9), 12741281 (2001)

[6] C. C. Yang, "Hybrid Wavelength-Division-Multiplexing/ SpectralAmplitude-Coding Optical CDMA System," IEEE Photonic Tech L. 17(6), 1343-1345 (2005).

[7] J. Huang, Y. Chang, and C. Hsu, "Hybrid WDM and optical CDMA implemented over waveguide-grating-based fiber-to-the-home networks," Opt. Fiber Technol. 13(3), 215-225 (2007).

[8] E. D. J. Smith, R. J. Blaikie, and D. P. Taylor, "Performance enhancement of spectral-amplitude coding optical CDMA using pulseposition modulation," IEEE Trans. Commun., vol. 46, pp. 1176-1185, Sept.1998.

[9] Yao-Tang Chang, Chuan-Ching Sue and Jen-Fa Huang, "Robust design for reconfigurable coder/decoders to protect against eavesdropping in spectral amplitude coding optical CDMA networks". J. Lightwave Technol. 25(8), 1931-1948 (2007).

[10] Govind P. Agrawal. Fiber-Optic Communications Systems, Third Edition, 2002 John Wiley \& Sons, Inc. ISBNs: 0-471-21571-6 (Hardback); 0-471-22114-7 (Electronic). Pp. (330-339). 